OPEN

SUBJECT AREAS:

COMPLEX NETWORKS

STATISTICAL PHYSICS

THERMODYNAMICS

STATISTICS

Received

8 April 2013

Accepted

8 November 2013

Published

25 November 2013

Correspondence and requests for materials should be addressed to A.G. (a. gudmundsson@es. rhul.ac.uk)

\section{Entropy and order in urban street networks}

\author{
Agust Gudmundsson' \& Nahid Mohajeri ${ }^{2,3}$
}

'Department of Earth Sciences, University of London Royal Holloway, Egham TW20 OEX, UK, ${ }^{2}$ Department of Geography, University College London, Gower Street, Pearson Building, London, WC1E 6BT, UK, ${ }^{3}$ Now at Solar Energy and Building Physics Laboratory (LESO-PB), Ecole Polytechnique Fédérale de Lausanne (EPFL), 1015 Lausanne, Switzerland.

Many complex networks erase parts of their geometry as they develop, so that their evolution is difficult to quantify and trace. Here we introduce entropy measures for quantifying the complexity of street orientations and length variations within planar networks and apply them to the street networks of 41 British cities, whose geometric evolution over centuries can be explored. The results show that the street networks of the old central parts of the cities have lower orientation/length entropies - the streets are more tightly ordered and form denser networks - than the outer and more recent parts. Entropy and street length increase, because of spreading, with distance from the network centre. Tracing the 400 -year evolution of one network indicates growth through densification (streets are added within the existing network) and expansion (streets are added at the margin of the network) and a gradual increase in entropy over time.

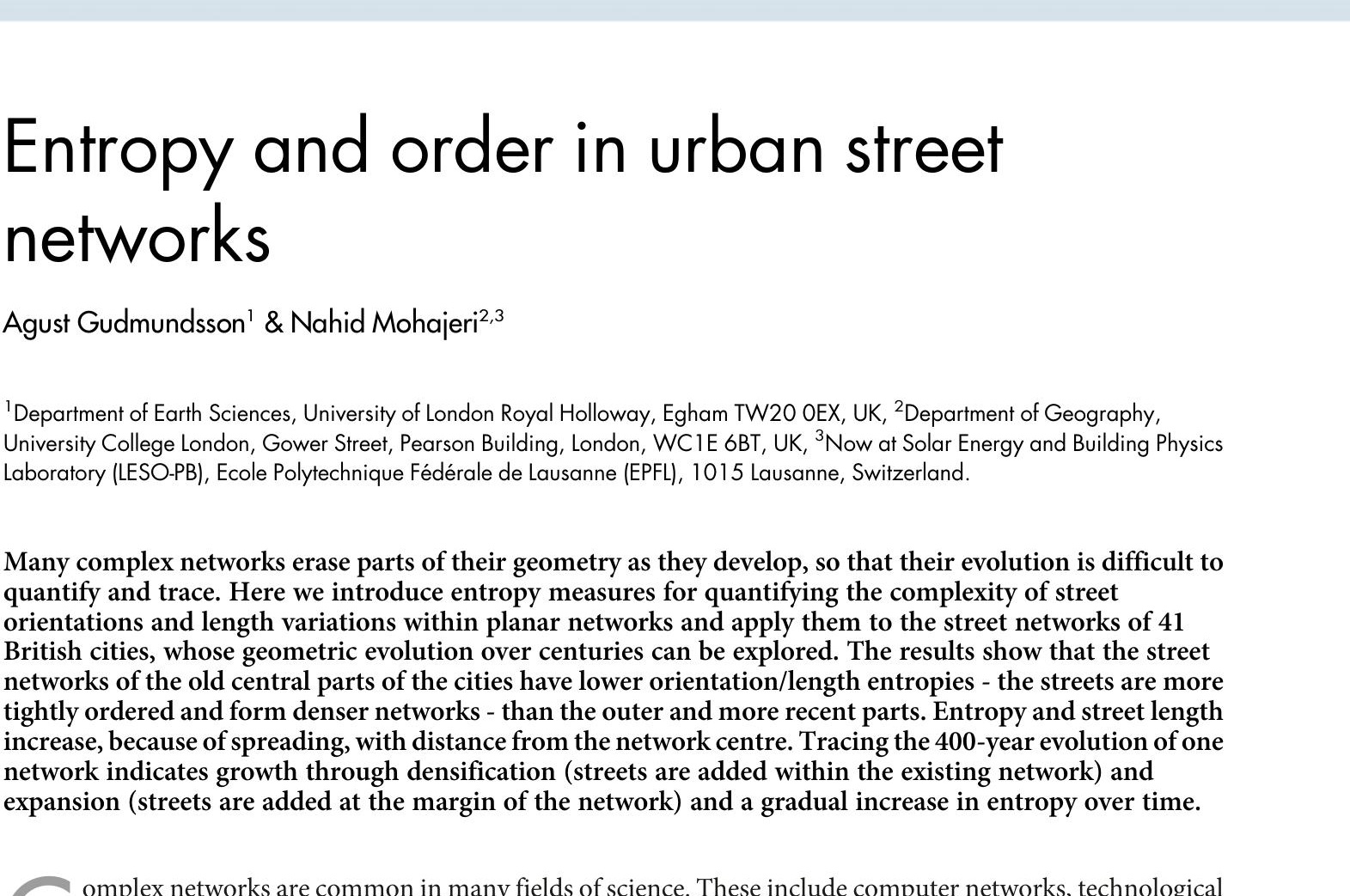

omplex networks are common in many fields of science. These include computer networks, technological networks, social networks, biological networks, and geological networks ${ }^{1-5}$. Many of these networks evolve in such a way that they partly or completely modify their geometric structure. This means that it is difficult - and for some networks impossible - to trace their history. In addition, many human-made networks have existed for comparatively short periods in comparison with the duration of the societies that made them.

By contrast, a street network preserves its history in the sense that a part of its geometry remains unchanged frozen in time - as the city evolves. Some street networks maintain their overall geometry for hundreds of years and are thus extremely resilient to change ${ }^{6,7}$. The resilience of many street networks makes it possible to study their long-term evolution as complex networks. Additional properties include, first, that street networks are essentially planar ${ }^{8}$; in the absence of tunnels and bridges, the streets (the links) cannot cross without generating an intersection or a junction, that is, a node. In the present paper, we define a street as the distance from one junction to the next one. Second, street networks are directional; they are composed of vectors. Each street has a magnitude, such as length, but also a direction (azimuth). Third, many street networks are subject to well-defined landform constraints as regards their evolution and overall geometry ${ }^{9,10}$. Fourth, street networks normally do not follow any general plans; they are to a large degree self-organised structures that respond to changes in internal and external forcing by gradual geometric changes.

There has been considerable work on street networks and urban growth using various methods ${ }^{4,8,11,12}$. Recently, the focus has been on the structural and topological properties of networks using different approaches ${ }^{8,13-18}$. By contrast, the orientations of streets and the network evolution have received comparatively little attention. Also, while entropy calculations have been used for trip distributions and related topics ${ }^{19,20}$, as well as to assess city heterogeneity ${ }^{17}$, the entropy method applied here has not previously been used to quantify orientation and length variations within and between evolving street networks.

The main aim of this paper is to present and analyse data on the street patterns of cities with a view of quantifying their complex-network characteristics. Particular attention is given to the geometric differences between the older (inner) and more recent (and outer) parts of the street networks. For analysing the networks, we introduce street orientation and street length entropy measures for quantifying these geometric differences. A second aim is to trace the 400-year evolution of the street network of the city of Dundee in Scotland and to relate the growth of the city to network densification, network expansion, and associated entropy changes.

\title{
Results
}

Orientations, lengths, and entropies of streets. To explore the street orientation, length, density, and network evolution, we analysed 41 British cities with a cumulative number of street segments of 753,322 (Fig. 1). The 


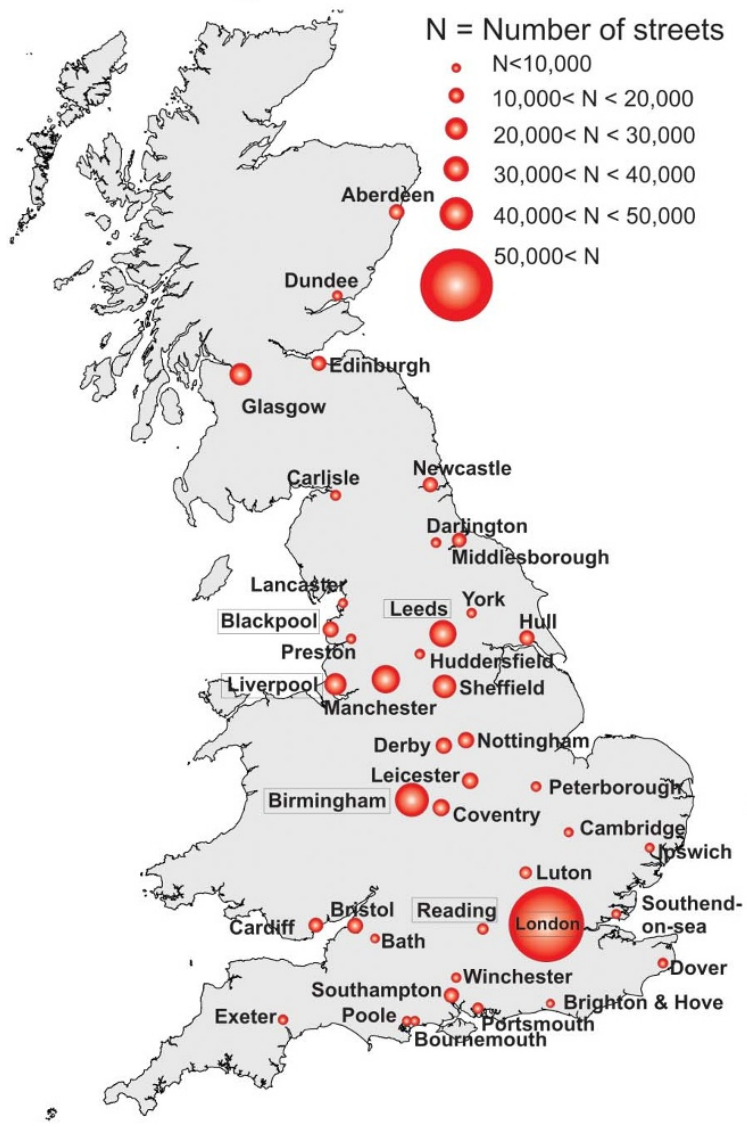

\begin{tabular}{cllll} 
b & c & d & e & $f$ \\
\hline $\begin{array}{l}\text { Orientation } \\
\text { distribution }\end{array}$ & $\begin{array}{l}\text { Number } \\
\text { of streets }\end{array}$ & $\begin{array}{l}\text { Orientation } \\
\text { entropy }\end{array}$ & $\begin{array}{l}\text { Rose } \\
\text { diagram }\end{array}$ & City \\
\hline 1000 Blackpool & & & & Ren
\end{tabular}

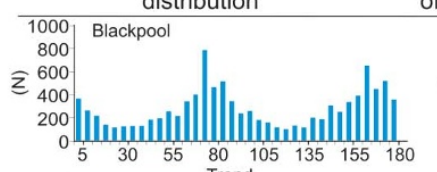

$\left.\begin{array}{r}1400 \\ 1200\end{array}\right]$ Liverpoo
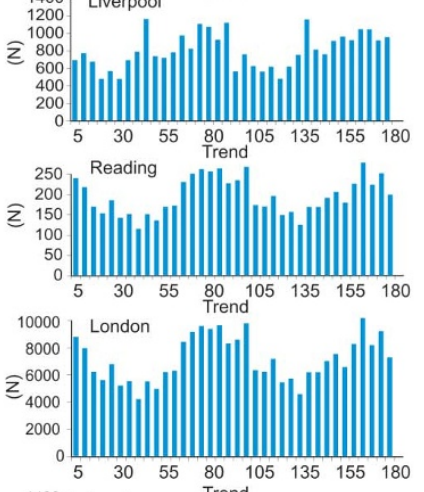

10077
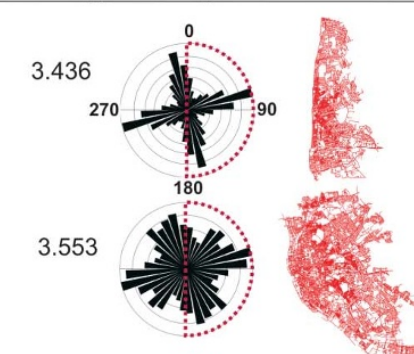

29050

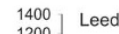

\begin{tabular}{r}
1400 \\
1200 \\
1000 \\
\hdashline \\
600 \\
400 \\
200
\end{tabular}

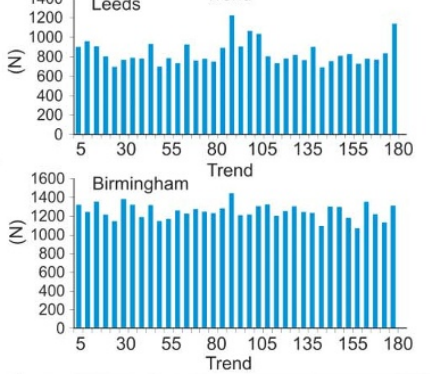

Figure 1 Location of the 41 British cities studied here. (a), City name, location, and its number of streets (source: OS Boundary-Line: District Borough Unitary Authority [Shape geospatial data], Coverage: Great Britain, Ordnance Survey (GB), Using: EDINA Digimap Boundary Download Service, $<$ http://edina.ac.uk/digimap >, Downloaded: September 2011). (b), Orientation histograms of 6 cities: Blackpool, Liverpool, Reading, London, Leeds, and Birmingham. (c), Number of streets. (d), Orientation entropy. (e), Rose diagrams. (f), The entire street network of the 6 cities. Map composed in ESRI ArcGIS 9.3.1.

selected cities range in population from just under 30 thousand to close to 8 million. Since they are all from the same country, various poorly-constrained cultural and climatic factors, which may differ widely between countries, do not significantly affect the network structures. There is no definitive agreement on how to define a city/street network boundary; here we use the administrative boundaries (and city populations) as determined by Office for National Statistics (www.nomisweb.co.uk). The street datasets were obtained from the Integrated Transport Network (ITN) layer and downloaded from the Edina Digimap website (www.edina.ac.uk).

As regards street orientations, all the cities fall between two extremes: Blackpool, with two roughly orthogonal sets generating a grid-like network (Figs. 1b, e), and Birmingham, with street orientation similar in all directions, generating a circular distribution (Figs. 1b,e). Six cites were selected to represent the general variation in orientation and related parameters (Fig. 1). To quantify better the differences in orientations and lengths of streets, within cities as well as between cities, we use the Gibbs/Shannon entropy formula, namely

$$
S=-k \sum_{i=1}^{t} p_{i} \ln p_{i}
$$

where $S$ is entropy, $k$ is a positive constant, $t$ is the number of bins with nonzero probabilities of streets, and $p_{i}$ is the probability of streets falling in the $\mathrm{i}$-th bin. For Gibbs entropy, $k$ is the Boltzmann constant, $k_{B}=1.38 \times 10^{-23} \mathrm{JK}^{-1}$. Here, however, the calculated entropies are dimensionless and given in the units of nat, which corresponds to $k_{B}$ being normalised to a factor of 1 . Eq. (1) is a perfectly general relation between entropy $S$ and probability $p_{i}$ : it is equally valid for equilibrium and non-equilibrium systems.

Theoretically, a street or a road can have essentially any length. In practice, the length is limited, for a city (or a town), to the diameter of the city, and for a country, to the diameter of the country, and so on. Furthermore, as defined here, a street is a segment that extends from one junction to the next, and is thus generally much shorter than these theoretical limits. The greater the number of streets per unit area of a city, the greater will be the street-network density and the shorter the average street length. Within these limits of street-length definition, there are no restrictions as such on the values that the street lengths can take (in a low-density network), and the same applies to the associated number of bins.

By contrast, the orientation of lineaments of various types can only take values between 0 and $360^{\circ}$. Furthermore, streets, and many geological lineaments (such as rock fractures), are oriented data, which means that they have no directional distinction and yield bidirectional or symmetric roses (Fig. 1e; cf. the section on Methods). It follows that the opposite classes or sectors $\left(180^{\circ}\right.$ apart) have the same street frequency, so that, for streets, the orientation can only take values between 0 and $180^{\circ}$. Since the class limits or bin widths for street orientation are here taken as $5^{\circ}$ (the same for all the street networks), the number of bins for the orientation entropy is fixed at 36 (Fig. 1b). The limited number of bins puts constraints on the possible variation in orientation entropy. 
The shape of the probability distribution affects its entropy. For example, if all the streets occupied a single bin the entropy would be zero. By contrast, if the distribution were uniform, so that all bins had the same lengths (heights), the entropy would reach its maximum value - given by the Boltzmann entropy equation, namely

$$
S=k \ln t
$$

It follows that Birmingham, with a close-to uniform distribution, has a higher entropy than Blackpool, with a peaked distribution (Figs. 1b, $\mathrm{d}, \mathrm{e})$.

The street length-size distributions of all the networks analysed here are heavy-tailed and follow approximately power laws. We tested how well power laws fitted the distributions in comparison with other functions using the maximum likelihood method. The results indicate a generally good power-law fit (see Table 1 and the discussion in the section on Methods).

For each network, we analysed three street sets: (a) all the streets of the city network, (b) the streets of the inner (older) or central part of the network and, for comparison, (c) the streets of an outer (recent) part of the network. The outer part was selected (1) to be as far from the centre of the network as possible, and (2) to have a very similar number of streets to that of the compared inner part. The inner part of the network is defined primarily by its being older and in most cases surrounded by a 'ring road' (a beltway or loop).

Correlations. The results show strong linear correlations between the length-entropies of the entire street sets and the street density (Fig. 2a) and the average street length (Fig. 2b). There are also correlations between the entropies of the outer and inner parts of the networks, calculated separately, and the density (Fig. 2c) and the average street length (Fig. 2d).

The negative linear correlation between entropy and density means that as the number of streets per unit area increases, the entropy decreases (Figs. 2a, c). Thus, the more confined the network, other things being equal, the lower is its entropy - in agreement with well-known thermodynamic effects of constraints ${ }^{21}$. By contrast, as the average length of the streets increases, the network expands and the entropy increases (Figs. 2b, d).

The entropies of the inner parts of all the networks (except for the city of Peterborough where major streets penetrate the centre) are lower than those of the outer parts (Fig. 2e). Similarly, for almost all the networks the average street length (Fig. 2f) and length range (Fig. 2h) are lower, and the density thus higher (Fig. 2f), in the inner than in the outer parts of the networks. These results are an indication of spreading in the lengths of the streets as the network grows. When the city becomes larger, the average distance between the streets, and thus their average length, increases (Figs. 2d, g). However, because the suburbs contain numerous residential areas, short streets continue to be constructed, so that the minimum length (commonly 3-5 m) stays more or less constant as the network expands while the average and maximum lengths increase. Consequently, the length range, which is the difference between the maximum and the minimum street length, also increases as the network expands (Fig. 2h).

Change in entropy with network growth. The change in entropy as a street network grows is illustrated further by the network of the city of Sheffield (Fig. 3). This is a medium-sized city, with a population of about 560,000 , a total number of streets of 23,500 , and a total cumulative street length of $1893 \mathrm{~km}$. Using 10 circles, covering a large fraction of the entire street network of Sheffield, the entropy was measured as a function of distance from the approximate network centre, defined as coinciding with the oldest part of the city. Each circle is made to include a similar number of streets (average 1500, range 1424-1568 streets). The results (Fig. 3) show a gradual increase in the entropy with distance from the network centre. The coefficient of determination $\mathrm{R}^{2}=0.7808$ implies that close to $80 \%$ in the variation in network entropy can be related to linear distance from the network centre.

For exploring the changes in network geometry and entropy during its growth, we traced the evolution of the street network of the city of Dundee, East Scotland (Fig. 1), for the past 400 years (Fig. 4). The evolution of the network of Dundee, which has a current population of 144,000 and 9,616 streets, is well documented and makes it possible to assess not only the entropy changes but also the way the network has grown during this period. The results show that, during these 400 years, the length entropy has gradually increased from 2.114 to 3.268 (Fig. 4a) while the orientation entropy has increased from 2.721 to 3.514 (Fig. 4b). The increase in length entropy coincides with a proportional increase in the tail part of the power-law length-size distribution (Fig. 4a), making the distribution more uniform. Similarly, the increase in orientation entropy relates to the orientation distribution becoming gradually more uniform (Fig. 4b).

As regards the network growth, two principal mechanisms operate at any particular time (Figs. 4c, d): (1) densification, that is, adding of streets within the existing network, and (2) expansion, that is, adding

Table 1 | Tests of power-law model for the street-network evolution of the city of Dundee. The symbols are as follows. Number of street segments for each time period ( $n$ ), scaling exponent based on MLE $(\alpha)$ ( \pm shows the standard error on $\alpha$ ), the number of observations in the power law range, ntail ( \pm shows the standard error of the ntaill, lower bound of power law $\left(x_{\text {min }}\right)$ at which the power law model no longer applies ( \pm shows the standard error of $x_{\min }$ ), power law fits and the corresponding p-values, and each of the subsequent rows shows the log likelihood ratios (LR) for the alternative models (fits) and the associated $p$-values. Positive values for LR indicate that the power law model is favoured over the alternative models if the $p$-value $<0.1$. However, if the $p$-value is large than 0.1 , the sign is not a reliable indicator as to which model provides the better fit to the data

Tests of power law behaviour in street data sets

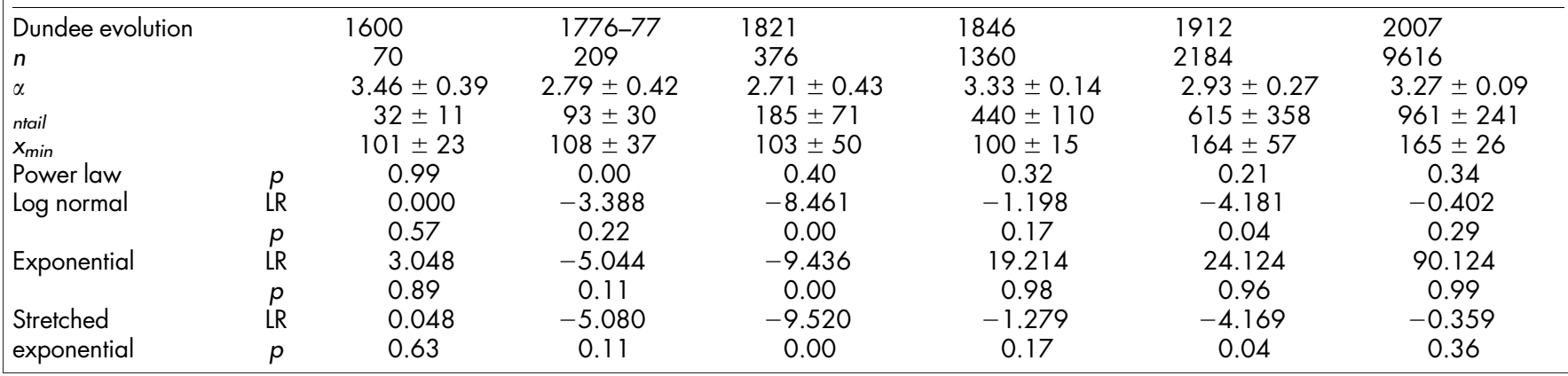



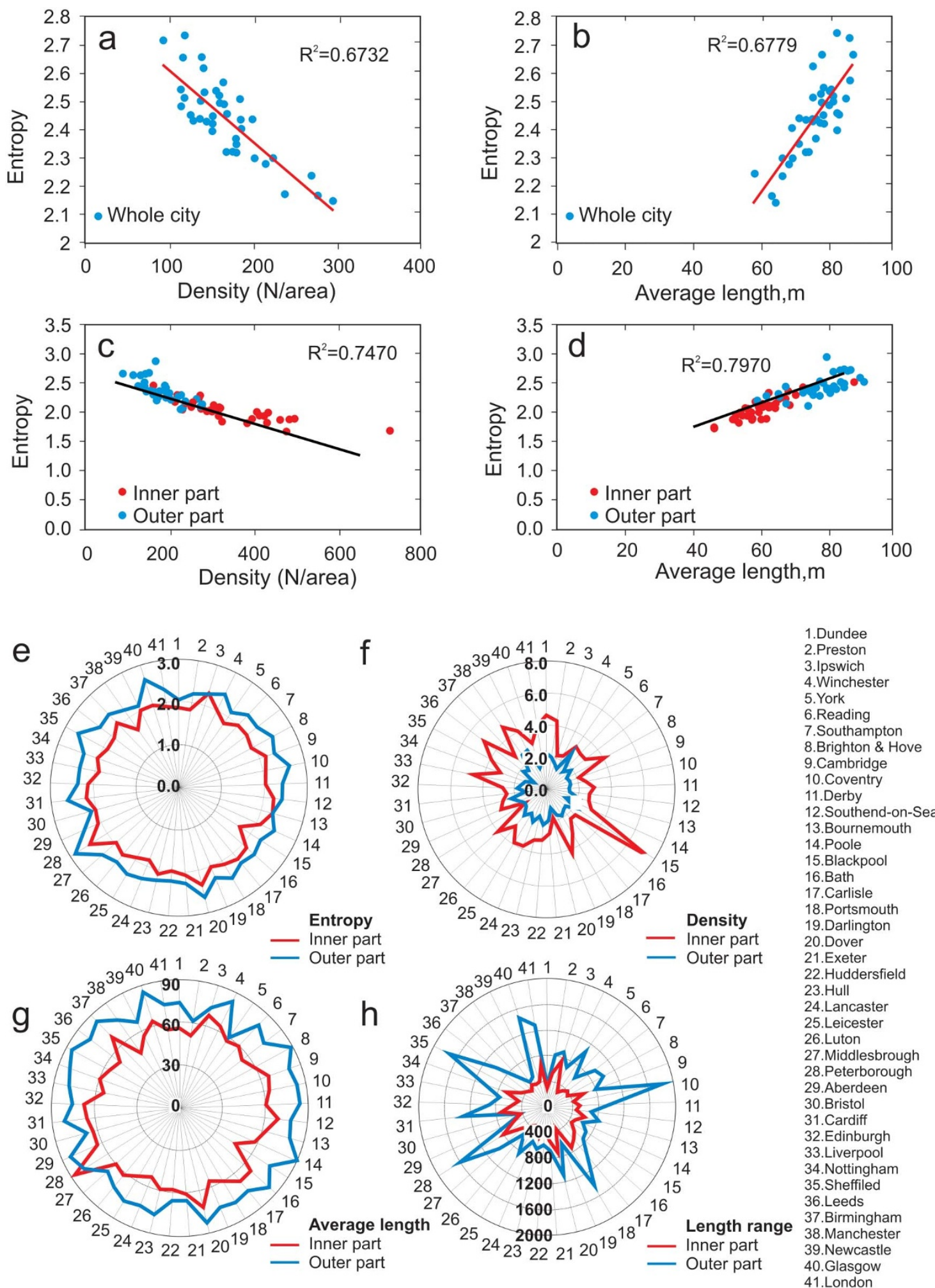

Figure 2 Street lengths and length entropies of the 41 networks. (a), Entropy versus density (number of streets per km²) for the networks of the whole cities. (b), Entropy versus average street length for the whole-city networks. (c), Entropy versus density for the inner (red dots) and outer (blue dots) parts of the networks. (d), Entropy versus average street length for the inner and outer parts of the networks. (e), Entropies of the inner (red) and outer (blue) parts of all the networks (the numbers refer to the named cities). (f), Network densities (numbers in hundreds) in the inner and outer parts. (g), Average street lengths $(\mathrm{m})$ in the inner and outer parts. $(\mathrm{h})$, Length range $(\mathrm{m})$ of streets in the inner and outer parts.

of streets at the margin of the network ${ }^{13}$. Densification is indicated when the maximum street length (the peak) in the recent year is less than that in the earlier year. These are marked by shaded zones, whereas the expansion is marked by white zones
(Fig. 4c). The figure shows that expansion dominates in the periods 1600-1776 and 1846-1912, whereas expansion and densification are more similar in the other three periods considered. For comparison, the entire street networks at these times are also 


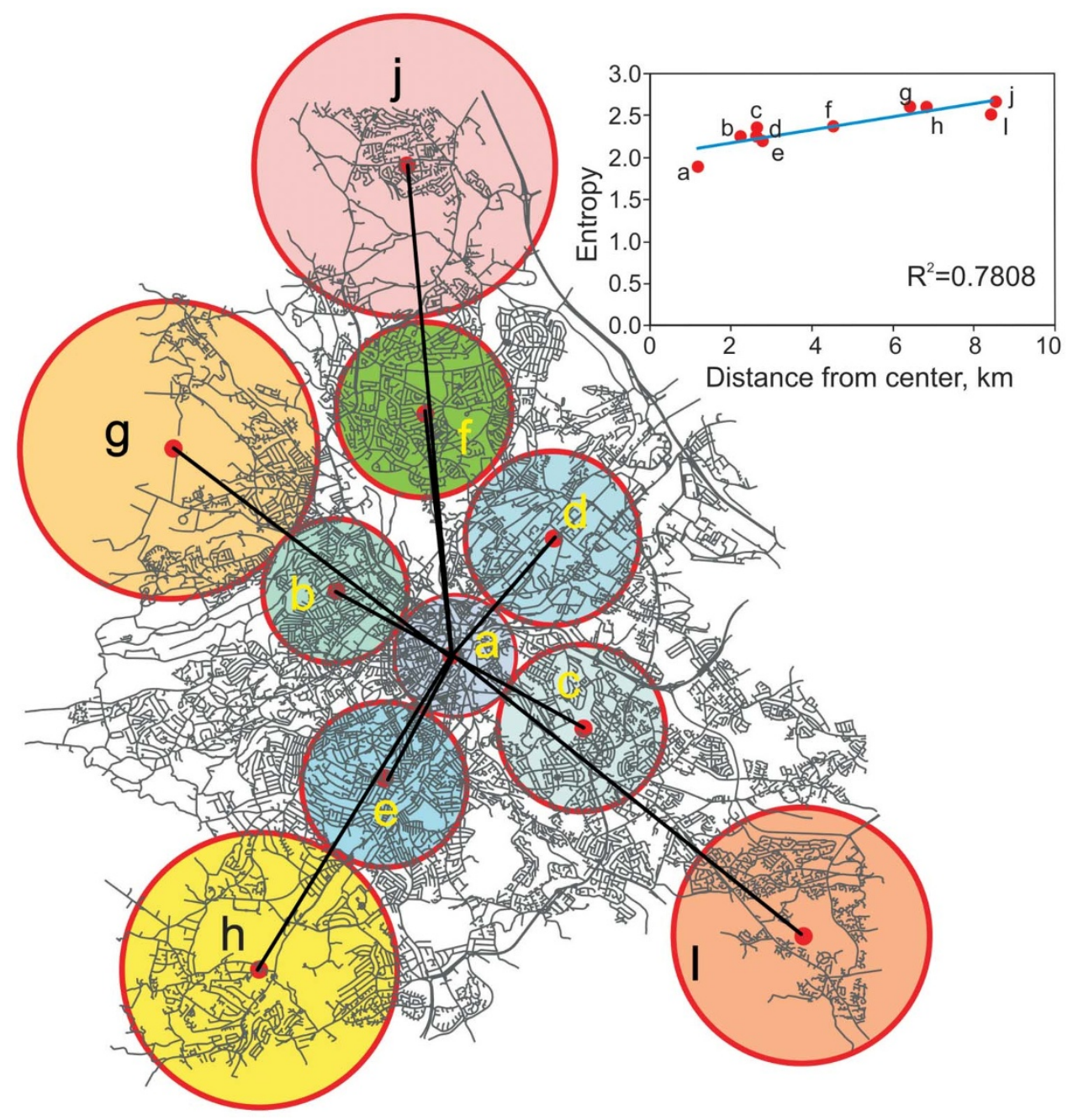

Figure 3 Variation in length entropy with distance from the centre of the street network of Sheffield. The circle in the centre, a, coincides with the oldest part of the network. (source: OS MasterMap ITN Layer [GML geospatial data], Coverage: UK, Ordnance Survey (GB), Using: EDINA Digimap Ordnance Survey Service, <http://edina.ac.uk/digimap>, Downloaded: September 2011). Map composed in ESRI ArcGIS 9.3.1.

shown (Fig. 4d). A detailed study has recently been made of the densification associated with the street (road) network of Milan ${ }^{13}$.

\section{Discussion}

Entropy is often related to 'disorder'. Perhaps a more appropriate description is to say that entropy is a measure of spreading or dispersal. From Eq. (1) it follows that entropy is the expectation value of the logarithm of the probability multiplied by a constant. In the present analysis the constant $k$ in Eqs. $(1,2)$ has the units of nat. As indicated above, this dimensionaless unit, used when the base of the logarithm in Eqs. $(1,2)$ is $e$, corresponds to the Boltzmann's constant $k_{B}$ being normalised to a factor 1 . By contrast, in statistical mechanics the corresponding Boltzmann's constant $k_{B}$ has the units of $\mathrm{J} \mathrm{K}^{-1}$. Clearly, the greater the spreading in the orientations of streets, the greater the entropy (Fig. 2); similarly, the greater the spreading in the lengths of streets, that is, the longer the tail of the power law, the greater is the entropy (Fig. 4). Since the number of bins for street orientation is here fixed at 36 , the limited number of bins puts constraints on the possible variation in orientation entropy.

The spread in street length is not constrained in the same way as the spread in orientation. As the tail of the power law becomes longer (Fig. 4), it follows that the spread in street length increases. For a constant bin width, the number of bins must increase with increasing tail length or spreading in length, and so does the entropy. A high range in street length also correlates with a high average length. The average length, in turn, is inversely related to the street-network density, as can be inferred from Fig. $(2 a, b)$. It follows that when the average street length of a network increases, so does the length entropy (Fig. 2). There have been several models proposed to explain why street networks tend to be denser (and thus with smaller average street lengths) in the inner parts of cities than in their outer parts (e.g $\mathrm{g}^{22}$.).

To test the correlation between entropy and street-network density further, we selected two inner-city parts of the same area $\left(1660 \mathrm{~km}^{2}\right)$ but with widely different street numbers. The inner parts selected are those of York, with 534 streets, and of Blackpool, with 1,194 streets. Thus, the number of streets in Blackpool, per unit area, is 2.2-times that of York. It follows that the average length of streets (the distance between street intersections) should be greater, and thus the entropy larger, in the inner part from York than from Blackpool. And that is exactly what is obtained: the average street length in York is $57 \mathrm{~m}$ (but $46 \mathrm{~m}$ in Blackpool), and the entropy of the inner part in York is 1.833 (but 1.685 in Blackpool). Thus, increasing (here more than doubling) the number of streets per unit area increases the street density, decreases the average street length, and decreases the entropy (Figs. 2a, 2c). Conversely, when the average street length increases - the streets become more spread - the entropy increases (Figs. 2b, 2d). This shows that for street (or other lineament) networks entropy is best interpreted as a measure of dispersal or spreading. 

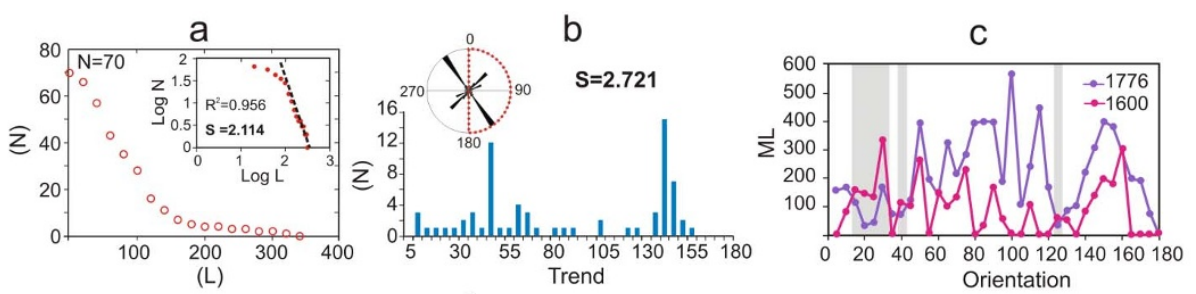

d
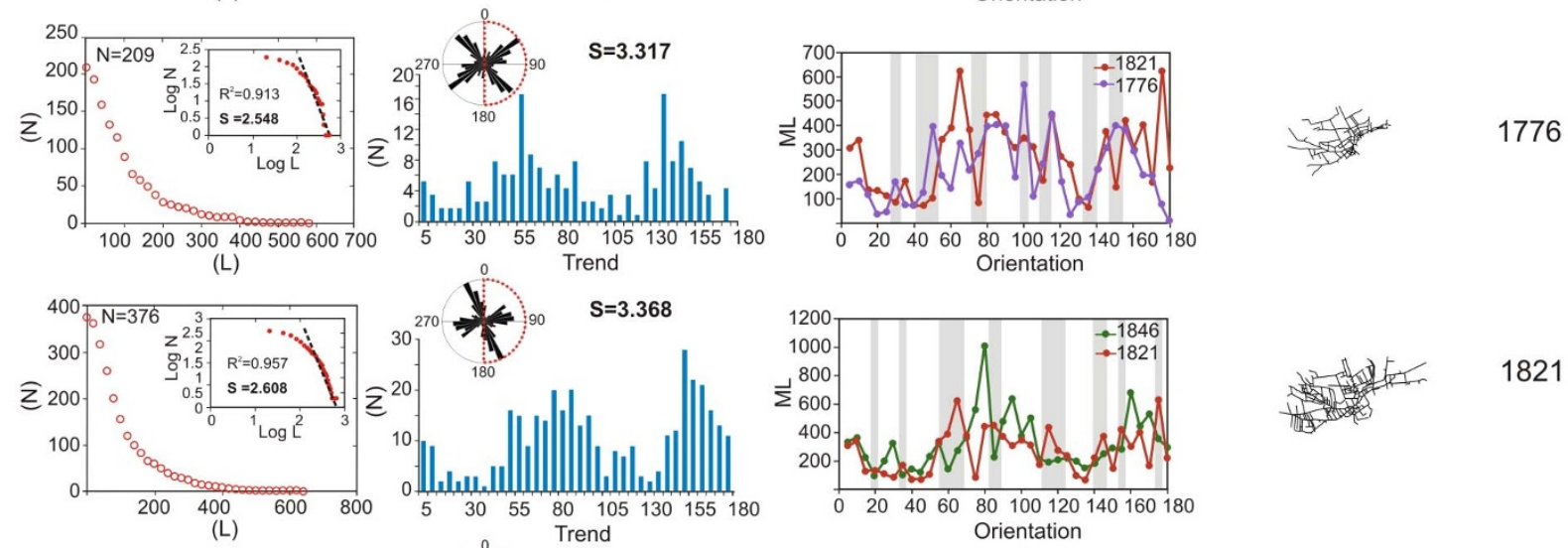

1821
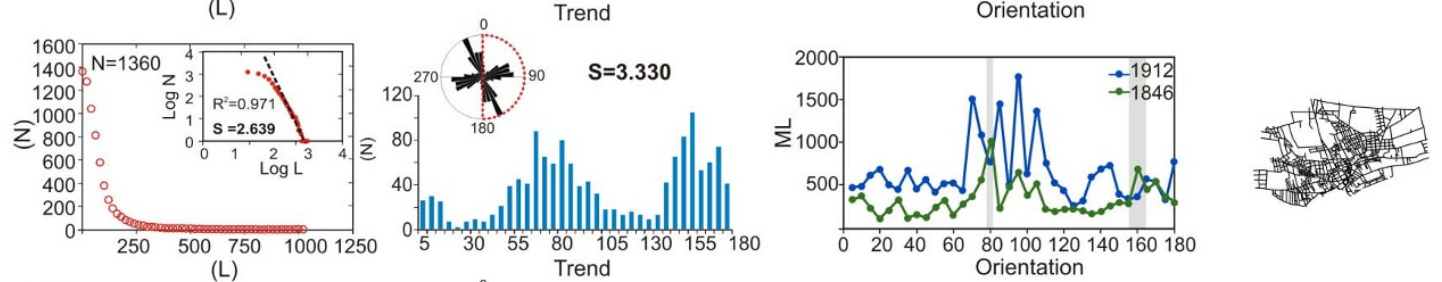

1846
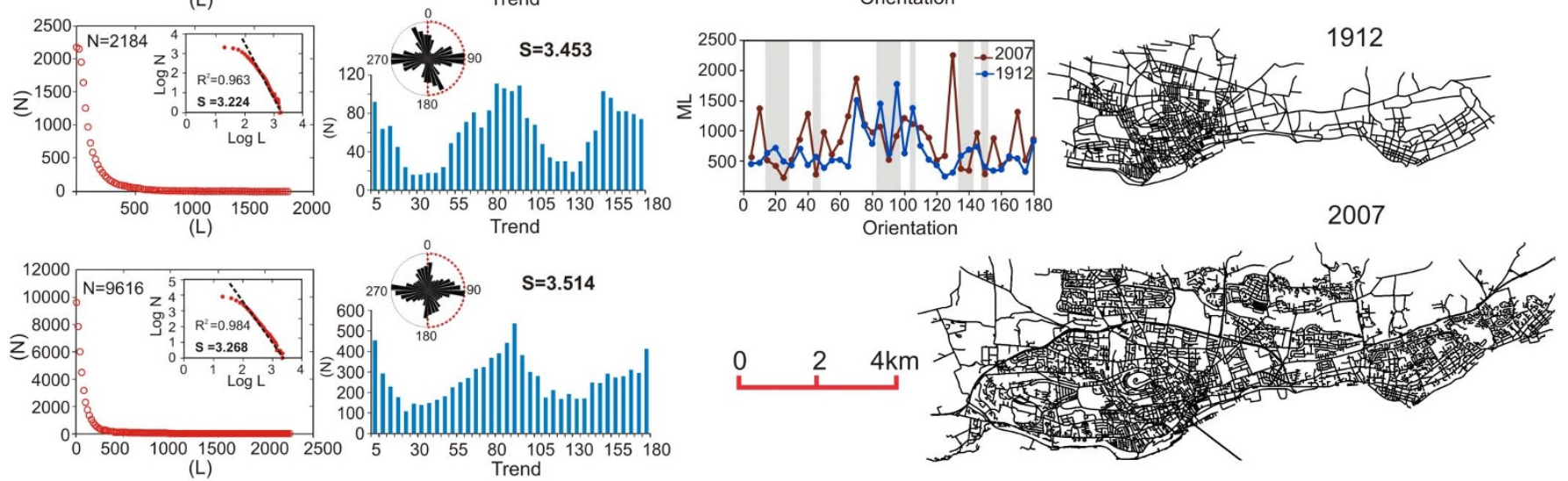

Figure $4 \mid$ Evolution of the street network of Dundee since AD 1600. (a), Power-law length distributions (inset: log-log plots and calculated tail entropies, S) for the time periods shown in d. L denotes street length in metres. (b), Orientation histograms, rose diagrams, and entropies, S. (c), Graphs showing maximum street length (ML) as a function of orientation. The shaded zones indicate the process of densification; the non-shaded (white) zones indicate the process of network expansion. (d), General evolution of the street network since 1600 and the reference times for the illustrations in a-c. (source: OS MasterMap ITN Layer [GML geospatial data], Coverage: UK, Ordnance Survey (GB), Using: EDINA Digimap Ordnance Survey Service, $<$ http://edina.ac.uk/digimap >, Downloaded: September 2011). Map composed in ESRI ArcGIS 9.3.1.

Street networks constitute a part of the wide field of complex networks. As is shown here, many street networks preserve their histories in the sense that a part of the network geometry remains essentially unchanged for hundreds of years. Their additional characteristics include that they are essentially planar and have geometric properties that can easily be measured and quantified. In spite of their size, accessibility, and geometric durability, street networks have received less attention than complex networks in many others fields ${ }^{1-5}$.

The present analysis of 41 street networks of British cities shows that these networks offer many interesting possibilities for geometric analysis and entropy studies that may be useful for comparison with other complex networks. The present real-world results are particularly relevant to the recently introduced methods for quantification of network complexity through entropy measures ${ }^{23,24}$. The orientation and length entropy results for the street networks are clearly useful for quantifying the geometric characteristics, as well as the evolution, of the present networks. We believe that the entropy measures and methods of visualisation introduced here may be used to analyse other networks, including their complex textural and structural variations and, where appropriate, their development through time.

\section{Methods}

It is well known that no definitive agreement exists as to how to define a city boundary. The definitions used depend much on the intended application ${ }^{25,26}$. In the present paper the city boundaries used are administrative boundaries; namely, those determined by Office for National Statistics (www.nomisweb.co.uk). The same source is used for the data on city populations. The datasets for the street networks were 

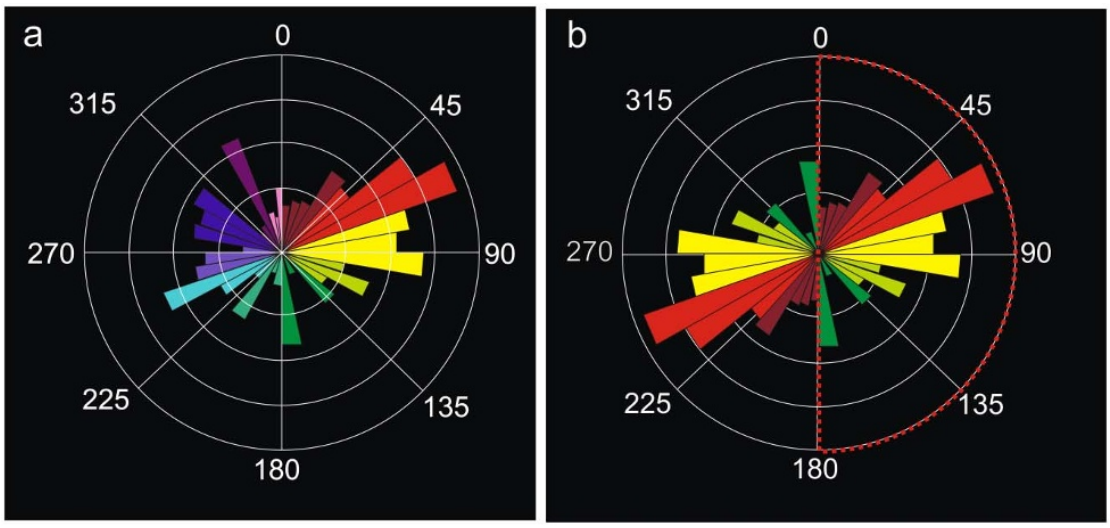

Figure 5 Rose diagrams of the frequencies of streets with different orientations (shown by different sectors). These diagrams serve to illustrate general circular-data presentations using rose diagrams; they are not based on data from the present paper. (a) Rose diagram showing a directional or asymmetric orientation distribution. (b) Rose diagram showing a bidirectional or symmetric orientation distribution. The eastern half circle (indicated by a red, broken line) spans $180^{\circ}$ and represents a semi-circle rose diagram (www.holcombecoughlinoliver.com).

obtained from the Integrated Transport Network (ITN) layer and downloaded from EDINA Digimap website (Digimap: www.edina.ac.uk). The ITN layer contains the street network and also transport information. Following this, the ITN layers were converted into GIS shape-file format.

The City Council of Dundee and the National Library of Scotland provided the historical maps for the city of Dundee (Fig. 4). All these historical maps were georeferenced and digitised in ArcGIS (ArcMap Version 9.3) after the maps had been imported into a GIS environment as a tif extension. Also, all the layers were projected using the coordinate system of the British National Grid and Transverse Mercator Projection. To align the different historical maps (geo-referencing) we used the historical buildings and the main streets as the reference points on the images. The street networks were drawn in vector format on the geo-referenced historical maps in an ArcGIS environment. Following this, the Network Analyst was used to build the entire network dataset for each historical map.

In the present analysis, intersections of streets are regarded as nodes and the streets themselves are regarded as links between nodes, that is, we use a primal representa$\operatorname{tion}^{27-30}$. Also, in the present study, the data is treated as an undirected network representation. This implies that each pair of nodes can only be connected to a maximum of one undirected link. It should be noted that, when analysing the geometry of a street network, either curved (real or physical) links or Euclidean (straightline) distances between the starting and end points of each street can be used. In the Euclidean analysis, all links are assumed to be straight lines. This assumption generates a systematic bias towards shorter link lengths ${ }^{8}$. In the present analysis we use physical links so that curved links are treated as curved when measuring their lengths.

For presenting the orientation of streets, we use rose diagrams (Figs. 1e, 4b). A rose diagram (Fig. 5) shows the frequencies of lineaments ${ }^{31}$, such as streets ${ }^{10}$ and geological fractures $^{32}$, and processes, such as the wind direction at a certain locality over a certain period of time (a wind rose). The standard rose diagrams are of two main types: (1) complete circles, which is the type used in the present paper, and (2) half circles (semicircles). More specifically, the orientation of each sector shows the orientation of streets and the length of the sector shows the number or frequency of streets of that orientation. For the present analysis of street orientations the program GEOrient (www.holcombecoughlinoliver.com) is used.

Both directional and oriented data can be used when analysing lineament orientation. In directional data we can distinguish one end of the lineament from the other or, alternatively, left from the right, as is the case for water flow in a river or for a dominating wind direction. By contrast, in oriented data there is no directional distinction, as is the case for geological fractures ${ }^{32}$ and streets in a city ${ }^{33}$. When the data are directional the rose diagram shows a unidirectional or asymmetric trend distribution (Fig. 5a). By contrast, when the data are oriented the rose diagram shows a bidirectional or symmetrical trend distribution (Fig. 5b). For directional data the measured data azimuths range from 0 to $360^{\circ}$. For oriented data, however, the opposite directions, $180^{\circ}$ apart, are equivalent. It follows that the graphical portrayal should then either be restricted to one-half of a complete circle, or have a rotational symmetry so that opposite classes or sectors in the rose have the same frequency (Fig. $\mathrm{b}^{31}$ ). In this study, the data presented are oriented (streets) so that the rose diagrams show bidirectional or symmetric trend distribution on a complete circle (Figs. 1e, 4b).

We tested how well the power laws fitted the street-length distributions in comparison with other functions or models, primarily the log normal, exponential, and stretched exponential functions. The testing was made using the maximum likelihood method $^{34}$, focusing on whether the data for each of the city-evolution periods are consistent with power laws (Fig. 4a). The results (Table 1) indicate that most of the Dundee-evolution data sets are consistent with power-law models, the one doubtful period being $1776-77$ where the $p$-value is 0.00 and thus less than 0.1 . However, $\mathrm{p}$-values for the alternative models are so large that we cannot decide which, if any, of the alternative models are statistically better than power laws. It should be noted, however, that even if the alternative models (log-normal, exponential and stretched exponentials) would give statistically better fits than power laws for some of the street network datasets, power-law fits would still be useful. This follows because different slopes (scaling exponents) of street-length distributions on log-log plots can often be used to distinguish between different street sets, that is, short and long streets which have different transport functionality ${ }^{10,33}$.

1. Caldarelli, G. Scale-free Networks: Complex Webs in Nature and Technology (Oxford University Press, Oxford, 2007).

2. Albert, R. \& Barabasi, A. Statistical mechanics of complex networks. Rev. Mod. Phys. 74, 47-97 (2002).

3. Newman, M. E. J. Networks: An Introduction (Oxford University Press, Oxford, 2005).

4. Barthelemy, M. Spatial networks. Phys. Reports 499, 1-101 (2011).

5. Estrada, E. The Structure of Complex Networks: Theory and Applications (Oxford University Press, Oxford, 2011).

6. Southworth, M. \& Ben-Joseph, E. Streets and the Shaping of Towns and Cities (Island Press, Washington D.C., 2003).

7. Marshall, S. Streets and Patterns (Spon Press, Abingdon, 2006).

8. Chan, S. H. Y., Donner, R. V. \& Lämmer, S. Urban road networks - spatial networks with universal geometric features? Eur. Phys. J. B. 84, 563-577 (2011).

9. Mohajeri, N. Effects of landscape constraints on street patterns in cities: examples from Khorramabad, Iran. Appl. Geogr. 34, 10-20 (2012).

10. Mohajeri, N. \& Gudmundsson, A. Entropies and scaling exponents of street and fracture networks. Entropy 14, 800-833 (2012).

11. Makse, H. A., Andrade, J. S., Batty, M., Havlin, S. \& Stanley, H. E. Modeling urban growth patterns with correlated percolation. Phys. Rev. E 58, 7054-7062 (1998).

12. Cardillo, A., Scellato, S., Latora, V. \& Porta, S. Structural properties of planar graphs of urban street patterns. Phys. Rev. E 73, 1-8 (2006).

13. Strano, E., Nicosia, V., Latora, V., Porta, S. \& Barthelemy, M. Elementary processes governing the evolution of road networks. Sci. Reports 2, 296 DOI: 10.1038/srep00296 (2012)

14. Jiang, B. A topological pattern of urban street networks: universality and peculiarity. Phys. A. 384, 647-655 (2007).

15. Barthelemy, M. \& Flammini, A. Modeling urban street patterns. Phys. Rev. Lett. 100, 138702 (2008)

16. Bettencourt, L. M. A., Lobo, J., Helbing, D., Kuehnert, C. \& West, G. B. Growth, innovation, scaling, and the pace of life in cities. Proc. Nat. Acad. Sci. 104, 7301-7306 (2007).

17. Levinson, D. Network structure and city size. Plos One 7, e29721 (2012).

18. Xie, F. \& Levinson, D. Measuring the structure of road networks. Geogr. Anal. 39, 336-356 (2007)

19. Wilson, A. G. Entropy in Urban and Regional Modelling (Pion, London, 1970).

20. Wilson, A. G. Complexity and Spatial Networks. Geggiani, A., Nijkamp, P. (eds.), 11-31 (Springer, Berlin, 2009).

21. Reiss, H. Methods of Thermodynamics (Dover, New York, 1996 reprint).

22. Levinson, D. \& Huang, A. A positive theory of network connectivity. Environ. Plan. B 39, 308-325 (2012).

23. Zhao, K., Halu, A., Severini, S. \& Bianconi, G. Entropy rate of non-equilibrium growing networks. Phys. Rev. E 84, 066113 (2011).

24. Zhao, K., Karsai, M. \& Bianconi, G. Entropy of dynamical social networks. Plos One 6, e28116 (2011).

25. Pont, M. B. \& Haupt, P. SpaceMatrix: Space, Density and Urban Form (NAi Publishers, Rotterdam, 2010).

26. Batty, M. \& Ferguson, P. Defining city size. Environ. Plan. B 38, 753-756 (2011). 
27. Jiang, B. \& Claramunt, C. A structural approach to the model generalization of an urban street network. GeoInformatica 8, 157-171 (2004a).

28. Scellato, S., Cardillo, A., Latora, V. \& Porta, S. The backbone of a city. Eur. Phys. J. B 50, 221-225 (2006).

29. Masucci, A. P., Smith, D., Crooks, A. \& Batty, M. Random planar graphs and the London street network. Eur. Phys. J. B 71, 259-271 (2009).

30. Gastner, M. T. \& Newman, M. E. J. The spatial structure of networks. Eur. Phys. J. B 49, 247-252 (2006).

31. Swan, A. R. H. \& Sandilands, M. Introduction to Geological Data Analysis (Blackwell, Oxford, 1995).

32. Gudmundsson, A. Rock Fractures in Geological Processes (Cambridge University Press, Cambridge, 2011).

33. Mohajeri, M., French, J. R. \& Batty, M. Evolution and entropy in the organization of urban street patterns. Annals of GIS 19, 1-16 (2013).

34. Clauset, A., Shalizi, C. \& Newman, M. E. J. Power-law distributions in empirical data. SIAM Rev. 51, 661-703 (2009).

\section{Acknowledgments}

We thank Edina, through UCL, for access to data.

\section{Author contributions}

N.M. designed and did the research, analysed the data, and made the calculations. A.G. and N.M. wrote and revised the manuscript.

\section{Additional information}

Competing financial interests: The authors declare no competing financial interests.

How to cite this article: Gudmundsson, A. \& Mohajeri, N. Entropy and order in urban street networks. Sci. Rep. 3, 3324; DOI:10.1038/srep03324 (2013).

(c) (1) (2) This work is licensed under a Creative Commons Attribution-

NonCommercial-ShareAlike 3.0 Unported license. To view a copy of this license, visit http://creativecommons.org/licenses/by-nc-sa/3.0 